\title{
Laparoscopic management of an adrenal cyst - A case report
}

\author{
Prashanth $\mathrm{AT}^{1}$, Krish Lakshman ${ }^{2}$ \\ ${ }^{1}$ DEPARTMENT OF GENERAL SURGERY, SAGAR HOSPITAL, \#44/54, 30TH CROSS, JAYANAGAR, BANGALORE, KARNATAKA, INDIA \\ ${ }^{2}$ SHANTHI HOSPITAL AND RESEARCH CENTRE (SHRC), 307, 40TH CROSS, 8TH BLOCK, JAYANAGAR, BANGALORE, INDIA
}

\begin{abstract}
AB STR ACT
Adrenal cysts are generally rare clinical entity, but due to advances in imaging diagnostic methods, detection incidental cases are increasing. Therefore, discussions about the therapeutic management of these cases are more numerous, due to the growing number of cases. We present a case of a 29-year-old woman who has had complaints of headaches and right low back pain in the last week. She had a history of high blood pressure in the past 6 months. Clinical examination was essentially normal. Routine blood investigations including a workup for pheochromocytoma was normal. CT abdomen and pelvis showed $8 \times 8 \times 8 \mathrm{~cm}$ cyst in the right adrenal gland. We decided to perform a laparoscopic excision of the adrenal cyst and successfully excised the adrenal cyst, fully saving the adrenal gland. Laparoscopic excision of the adrenal cyst seems to be the best recommended treatment option for these pathologies. The probability of recurrence after surgical excision is low.
\end{abstract}

\author{
Category: Case Presentation \\ Received: July 18, 2020 \\ Accepted: August 23, 2020 \\ Keywords: \\ adrenal cyst, laparoscopy, adrenal cyst excision \\ *Corresponding author: \\ Prashanth A T, \\ Department of General Surgery, Sagar Hospital, \#44/54, \\ 30th Cross, Jayanagar, Bangalore, Karnataka, India \\ E-mail: at.prashanth@gmail.com
}

\section{Introduction}

Adrenal cysts are generally rare clinical entities. In the modern era, most adrenal cysts are incidentally identified due to improved cross-sectional imaging modalities and also the increased frequency of imaging studies [1]. The incidence of adrenal cysts in the overall population is very low, below $0.2 \%$ value. Traditionally, adrenal cysts can be classified as functional or non-functional cysts, and histopathologically as benign or malignant. Non-functional adrenal cysts may be further classified as endothelial cysts $(45 \%)$, pseudocyst (39\%), epithelial $(9 \%)$ and parasitic cysts (7\%) [2].

Majority of the adrenal cysts are asymptomatic and less than $10 \mathrm{~cm}$ in diameter when detected incidentally. However, the problems like pain, gastrointestinal disturbances, intracystic bleeding or infection occur when the cysts become large. Some rare presentations include hypertension or spontaneous rupture of the cyst [3].

We report a case of adrenal cyst as presented with headache and hypertension. The laparoscopic excision of the adrenal cyst is described, which was our therapeutic option for the presented case.

\section{Case Presentation}

A 29-year-old female presented with complaints of headache and right loin pain in the past 1 week. The pain had an insidious onset, gradually progressive, nonradiating and with no aggravating / relieving factors. There were no associated symptoms such as fever, nausea, vomiting, or bowel and bladder disorders. There were no obvious manifestations of an endocrine disorder. From the anamnesis it was noted that the medical and surgical history was insignificant. At the general physical examination, the patient was afebrile, her BMI was 28 $\mathrm{kg} / \mathrm{m} 2$, and the blood pressure was $130 / 80 \mathrm{~mm} \mathrm{Hg}$.

The routine bloods tests were within normal limits. Investigations regarding pheochromocytoma did not reveal any abnormalities. CT abdomen and pelvis showed $8 \mathrm{~cm}$ thin-walled cyst in the right adrenal gland. Both the kidneys were normal in appearance on this examination (Figure 1).

We decided to perform a laparoscopic adrenal cyst excision. Patient was placed in a semi left lateral position. Four ports were used ( 2 ten $\mathrm{mm}$ and 2 five $\mathrm{mm}$ ). The camera port was 2 inches above the umbilicus in the 
midline, and the other three ports were triangulated. On examination, the rest of the peritoneal cavity was normal. The surgery started with the mobilization of hepatic flexure using the harmonic dissector. Inferior vena cava was visualized after duodenal kocherization. Gerota's fascia opened. The adrenal cyst arising from antero-superior surface of the gland was visualized. Decompression of the cyst was done. Around $100 \mathrm{ml}$ of straw color fluid was aspirated out. After dissecting all around, cyst was completely excised along with a small rim of adrenal tissue and specimen retrieved through the camera port. Hemostasis was maintained throughout the surgery. The procedure took 90 minutes for completion. Intraoperative pictures were seen in Figure 2. The patient had an uneventful recovery. Histopathology has shown that it is a hemorrhagic cyst.

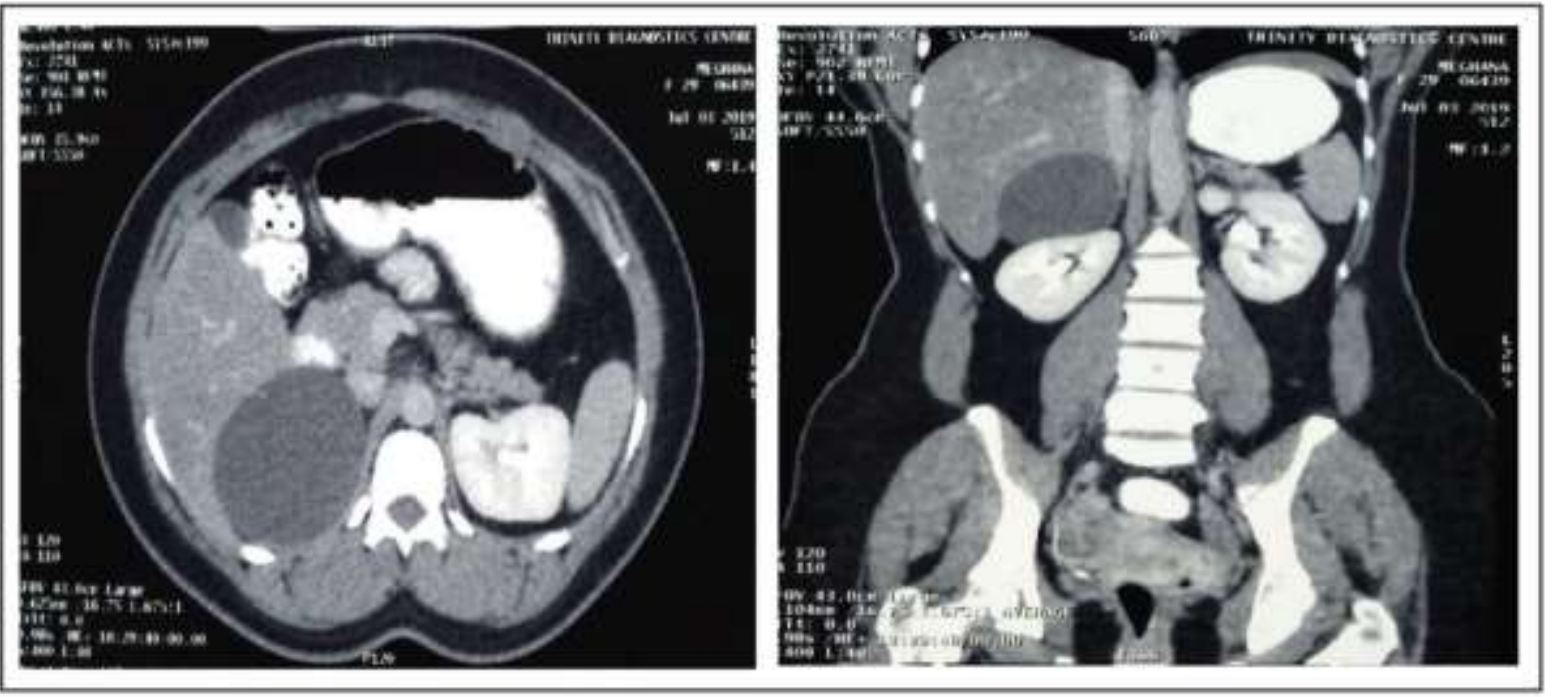

Figure 1. CT Abdomen and Pelvis
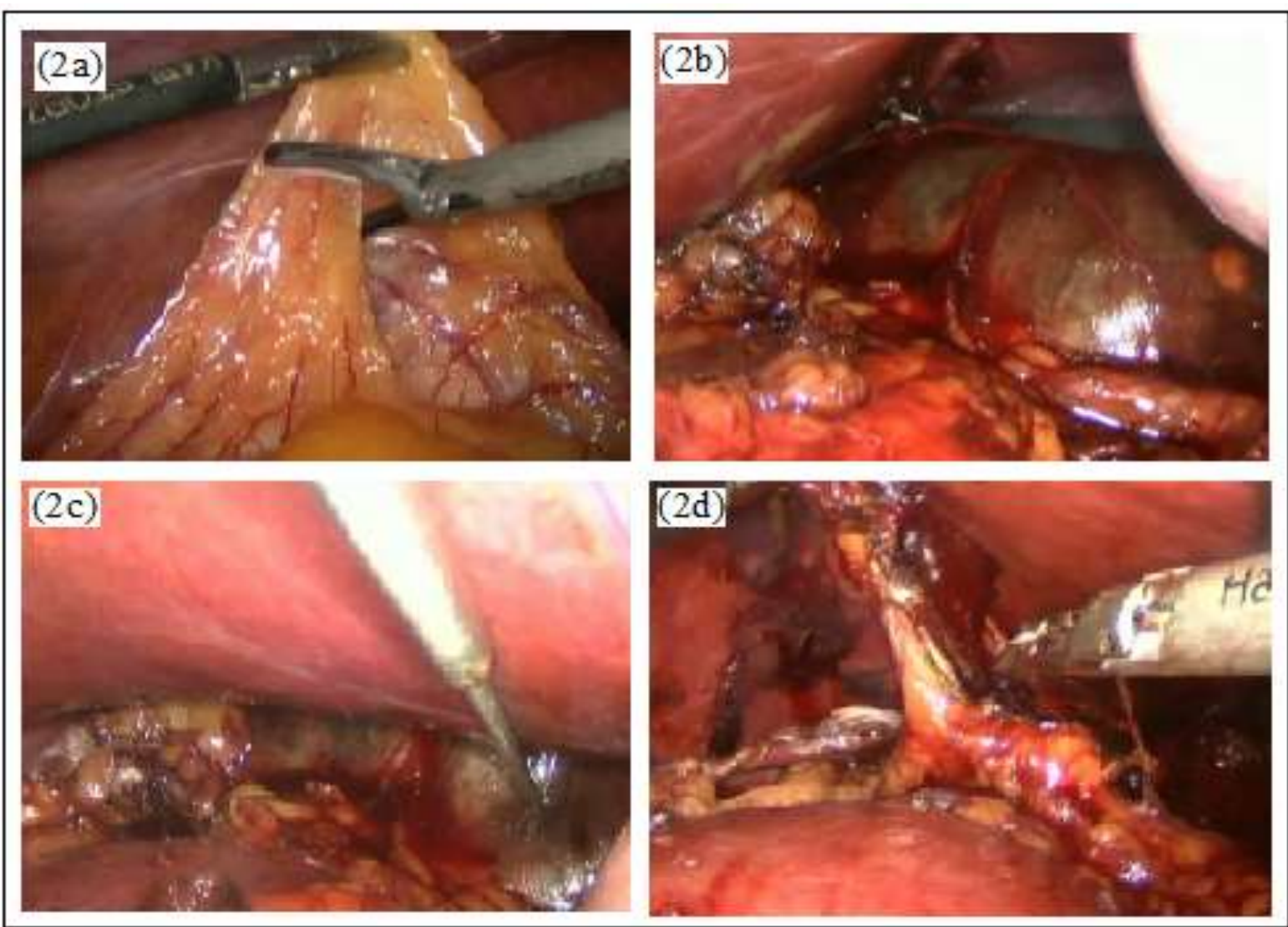

Figure 2. Intraoperative pictures at various stages of surgery; (2a) - removal of omental adhesions to the cyst; (2b) - defining the extent of the cyst; (2c) - aspiration of the cyst; (2d) - excision of the cyst sparing the adrenal gland. 


\section{Discussions}

Cysts of the adrenal gland in the overall population is very low, but nowadays with the increasing application of imaging modalities more cysts are being detected incidentally. Adrenal cysts are usually asymptomatic but some cases may present with acute abdominal pain and can mimic an acute abdomen whenever bleeding occurs into the cyst. Very rarely adrenal cyst can present as huge abdominal mass causing discomfort and also some cysts were hormonally active [4].

A degree of controversy exists between conservative and surgical management of asymptomatic adrenal cysts. However, management strategy is influenced by (a) functional status of the cyst, (b) potential complications such as hemorrhage into the cyst and (c) the possibility of incidental malignancy [5].

Surgical management would be necessary in a functional lesion, potentially malignant lesion or benign lesion more than $5 \mathrm{~cm} \mathrm{[6].} \mathrm{For} \mathrm{smaller} \mathrm{cyst,} \mathrm{the} \mathrm{aspiration}$ has been one of the options, but recurrence remains a possible evolution. Several authors have described open surgical excision [4]. However, laparoscopic procedures have gained popularity and have become the procedure of choice. Laparoscopic procedures have a clear advantage over open surgery in terms of less pain, blood loss, complication rate and hospital stay. The advantages of the laparoscopic procedure have been demonstrated in several studies [7].

\section{Conclusions}

Even though adrenal cysts are rare clinical entity, many of them were being detected incidentally due to the wider use of imaging modalities. We recommend laparoscopic cyst excision for these cases because it is feasible, safe and has the advantage of minimal trauma, shorter hospital stay and less blood loss.

\section{Conflict of interest disclosure}

There are no known conflicts of interest in the publication of this article. The manuscript was read and approved by all authors.

\section{Compliance with ethical standards}

Any aspect of the work covered in this manuscript has been conducted with the ethical approval of all relevant bodies and that such approvals are acknowledged within the manuscript. The patient's permission to report this case was obtained.

\section{References}

1. Favorito LA, Lott FM, Cavalcante AG. Traumatic rupture of adrenal pseudocyst leading to massive hemorrhage in retroperitoneum. Int Braz J Urol. 2004;30(1):35-36.

doi:10.1590/s1677-55382004000100008

2. Guo YK, Yang ZG, Li Y, et al. Uncommon adrenal masses: CT and MRI features with histopathologic correlation. Eur J Radiol. 2007;62(3):359-370. doi:10.1016/j.ejrad.2006.12.011.

3. de Bree E, Schoretsanitis G, Melissas J, Christodoulakis M, Tsiftsis D. Cysts of the adrenal gland: diagnosis and management. Int Urol Nephrol. 1998;30(4):369-376. doi:10.1007/BF02550213

4. Poiana C, Carsote M, Chirita C, Terzea D, Paun S, Beuran M. Giant adrenal cyst: case study. J Med Life. 2010;3(3):308-313.

5. Chew SP, Sim R, Teoh TA, Low CH. Haemorrhage into non-functioning adrenal cysts--report of two cases and review of the literature. Ann Acad Med Singap. 1999;28(6):863-866.

6. Wedmid A, Palese M. Diagnosis and treatment of the adrenal cyst. Curr Urol Rep. 2010;11(1):44-50. doi:10.1007/s11934-009-0080-1

7. Castillo OA, Litvak JP, Kerkebe M, Urena RD. Laparoscopic management of symptomatic and large adrenal cysts. $J$ Urol. 2005;173(3):915-917. doi:10.1097/01.ju.0000152177.35204.70 